\title{
Compression of image using hybrid combination of haar wavelet transform and color space model in noisy environment
}

\author{
Sridhar C $\mathbf{S}^{1^{*}}$, Mahadevan.G ${ }^{2}$, S.K.Khadar Basha ${ }^{3}$, G.ChenchuKrishnaiah ${ }^{4}$ \\ ${ }^{1}$ Research scholar, Bharthiar University, Coimbatore, \\ ${ }^{2}$ Principal, ANNAI College, Kumbakonam, \\ ${ }^{3}$ Assistant Professor, SJCIT,Chickballapur, \\ ${ }^{4}$ Professor, GKEC, Sullurpet, Nellore, \\ *Corresponding author E-mail: sridhar_cs@yahoo.com
}

\begin{abstract}
This article manages the examination of image compression utilizing crossover blend of both wavelet change and color space.. The paper covers foundation which incorporates decay of image, thresholding in wavelet for image compression. The compressed image of Haar wavelet is then gone through color space transformation which is utilized to change the color image into grey scale image. On transformation to grey scale image, the number of bits and transfer speed gets diminished. It is conceivable to process the images of various organizations inside the security issues that have been additionally discussed and actualized. The outcomes are shown as depictions in the results.
\end{abstract}

Keywords: Compression,Haar Transform, Image Processing, Thresholding.

\section{Introduction}

Compression assumes an awesome part in preparing of still or moving images. It's significance is connected in various fields of signal handling. It is a procedure of decreasing the measure of information required to speak to a specific measure of data by expelling the excess inside the information. Discrete Cosine Transform (DCT) filters alone from the image specifying by parts of outset frequencies. Not withstanding, progression quantization, with compression ordinarily happens for thresholding, the minimum critical frequencies are disposed of, subsequently the utilization of the term of "lossy"[3]. Therefore, the recreated image contains some distortion that can be balanced amid the compression stage.

Consequently, it is critical to compress the image by putting away just the basic data expected to reconstruct.any image under consideration can be just analysed as the tight fit of pixels and its values.hence for the process of compression,the pixel regions without much similarity has to be minimized.Redundancies are showing up in the regions of uniform shading, and on the other images that have continuous and extensive changes in shading may not get compressed. A total orthogonal arrangement are characterized by wavelet of Haar [4].

This arrangement of functions has a property that each constant function on interim $[0,1]$ might be spoken to by a consistently and merged arrangement as far as components of this framework. These days, in the literature, there are some different views of the Haar functions [5]. Those definitions are commonly varying as for the estimations of Haar capacities at the purposes of discontinuity

\section{System model}

The various stages of an wavelet transformation are entropy coding,transformation and quantization as represented in figure 1. To build the decoder, exactly vice versa process of encoder has been followed without much modification. The decoding process is obtained by an inverse transform in order to approximate the original image [6]

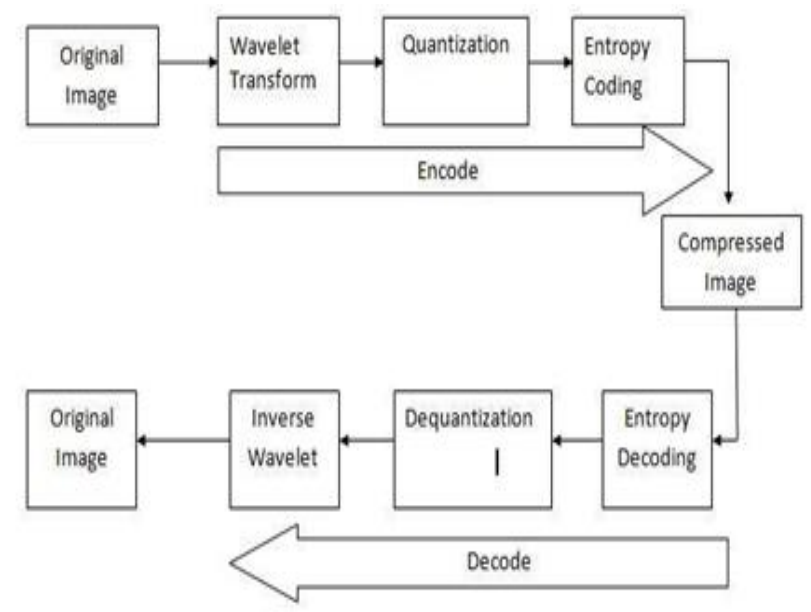

Fig. 1: Block diagram of encode and decode process by using wavelet transformation algorithm 


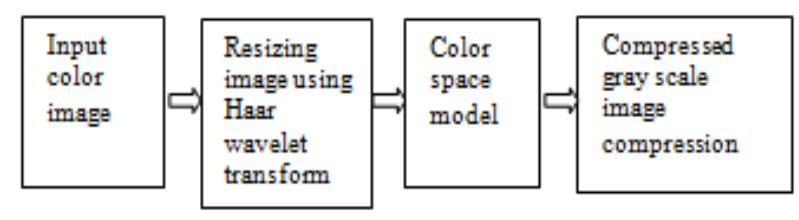

Fig. 2: Block diagram of color to gray process by using color space model and Haar wavelet transform

The first image is connected as information image to the Wavelet for resizing .After decomposition, quantization is required for threading and entropy coding is done to acquire the compressed image. reconsturcted image is retreived utilizing reverse strategy entropy decoding, dequantization and inverse wavelet change.

The unique property of our analysis is the energy preservation. The energy is oriented as the squares of the sum of the squares of the its value quantities. The energy of an image is the whole of the squares of the pixel esteems and the energy in the image is the aggregate of the squares of the change coefficients. Subsequently, the energy of a signal gets partitioned amongst estimate and subtle elements signals, however the aggregate energy continues as before. energy is lost inspite of thresholding as it changes the coefficient esteems and thus the compressed adaptation contains less energy. Compaction happens between the sizes of the detail coefficients that are essentially less than those of the estimate coefficients.

Quantization is a procedure of mapping an arrangement of ceaselessly esteemed information, $\mathrm{x}$, to an arrangement of discrete esteemed yield information, y [7]. Fig. 2 demonstrates the quantization procedure.

The quantized information contains repetitive data which can be overwhelmed by utilizing entropy encoding. The wavelet coding relies upon the likelihood that the coefficients of a change that style relates the pixels of a image can be coded more capably than the primary pixels themselves.

Generally, as a human, we may characterize the diverse hues by its luminance, shade, splendor and vividness. An individual may describe a color utilizing the measure of Red, Green and Blue, likewise, cyan, maroon, yellow, white and dark hues, which are the blending or mixes of the distinctive hues [4].

Distinctive between Red, Green and Blue color space has three channels and for the most part used to portray the images and PC illustrations. Hues like luminance, chrominance, power and shine shading space has three channels with various arrangements utilized as a part of video frameworks. Hues like CMYK are utilized as a part of printing frameworks.

Red, Green and Blue colors (RGB):

The Red, Green and Blue colors are for the most part used to depict the images and computer representations. These color space has three channels. These red, green and blue are essential shading segments that are included to get wanted color like cyan, maroon, yellow, white and dark colors.

Luminance and Chrominance ( $\mathrm{YCrCb})$ :

The luminance and chrominance hues are for the most part utilized as a part of video frameworks. This shading space has three channels. These YUV hues has diverse organizations to depict shading images and dim images. Distinctive YUV positions are YUV444, YUV420[3]. In these organizations Y remains for the luma part (shine), $\mathrm{Cr}$ and $\mathrm{Cb}$ are the chrominance (shading) segments. These $\mathrm{Cr}$ and $\mathrm{Cb}$ chrominance hues are picked as Red and Blue colors[5].

The YUV444 information design transmits 24 bits for each pixel. Every pixel is appointed special Y, $\mathrm{U}$ and $\mathrm{V}$ estimations of 1 byte for each esteem. The bytes are requested in the image in the accompanying way, where $\mathrm{Y}, \mathrm{U}, \mathrm{V}$ speak to a solitary byte of shading or brilliance esteem, and $0,1,2 \ldots$ speak to the pixel numbers related with those qualities: U0 Y0 V0 U1 Y1 V1 U2 Y2 $\mathrm{V} 2 \ldots$
The YUV422 information design shares U and V esteems between two pixels. Thus, these qualities are transmitted to the PC image cradle, once for each two pixels, bringing about a normal transmission rate of 16 bits for every pixel [4].

The bytes are ordered in the image in the following manner: U0 Y0 V0 Y1 U2 Y2 V2 Y3 U4 Y4 V4..

The inverse wavelet transform, dequantization and entropy decoding are the operations of wavelet transform, quantization and entropy encoding separately.

The work is especially focused towards wavelet image compression utilizing Haar Transformation with a plan to limit the computational prerequisites by applying diverse compression edges for the wavelet coefficients and these outcomes are acquired in division of seconds and therefore to enhance the nature of the reconstructed image

High compression rates and better image quality are analyzed for reproduced images. It likewise abuses the connection attributes of the wavelet coefficients and additionally second request qualities of images in the outline of enhanced lossy compression frameworks for medicinal and noisy images.

The various steps of decomposition is as shown in the figure 3 , Through discrete wavelet change, a signal can be poor around doing it through an examination channel bank took after by a devastation operation. This examination channel bank, which includes a low pass and a high pass channel at each deterioration organize, is typically used as a piece of image. Right when a signal adheres to these procedures, it parts into two gatherings. The low pass channel, which looks at to an averaging operation, evacuates the coarse information of the signal. The yield of the isolating operations is then wrecked by two. A two-dimensional change can be master by performing two separate onedimensional changes.

For a couple of signals, an expansive number of the wavelet coefficients are close or comparable to zero. Thresholding can change the coefficients to convey more zeros. This should then make various consecutive zeros which can be secured in extensively less space, and transmitted more quickly by using entropy coding pressure. By then we got packed picture.

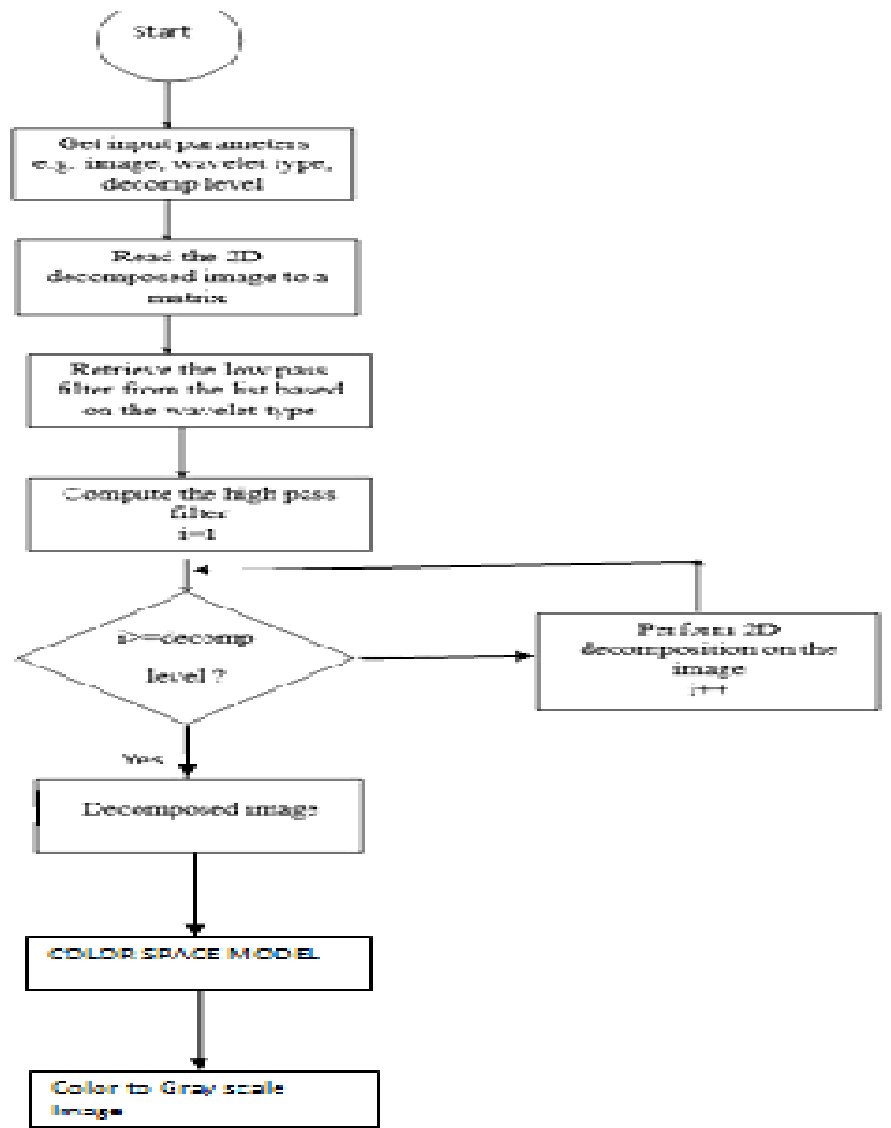

Fig. 3: flowchart for resizing and color to gray scale conversion 


\section{Result and analysis}

In the domain of image processing, Haar wavelet transformation has gained its nobel position by the utilization of four different filters namely two for decomposition,two for reconstruction namely high pass and low pass respectively.

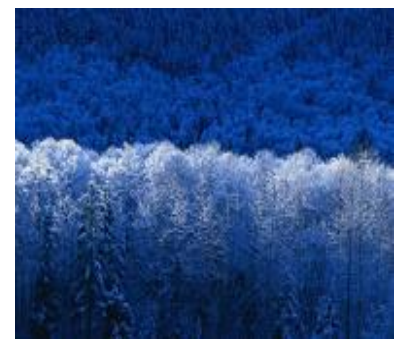

(a) Original image

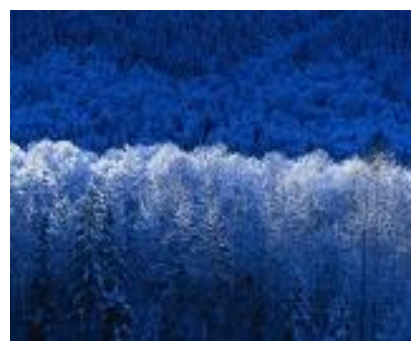

(b) Resizing of color image usig Haar Wavelets

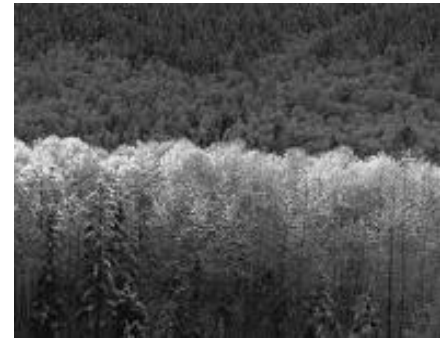

(c) Compressd grey image using color space model

Fig. 4: Images of our model at various stages

Initially let us consider an unique image, the image under consideration is decomposed by Haar wavelets in regard of low pass, high pass channels for deterioration and disintegration respectively. By decomposition through edge esteem, we dispense with every single excess datum. Resizing of image is finished. This resized image is then gone through shading space show which additionally changes over resized image into compressed dim scale image.

To acquire a reconstructed image at the less than desirable end, we first change over grey scale image into color image. At this point of consideration the resizing is completed by involving both the types of filters viz., low pass and high pass corrospondant to HAAR Wavelet.It is conceivable to reconstruct a unique image from the compressed image Fig. 4.

The table I depicts the image total size with respect to original image and other processed images with the techniques discussed

Table 1: The reconstructed Image and original image size

\begin{tabular}{cccc}
\hline Image & Original & HAAR Compressed & Color space model \\
\hline & image & Color image & Gray scale image \\
Size & $208 \mathrm{~KB}$ & $72 \mathrm{~KB}$ & $63 \mathrm{~KB}$ \\
\hline
\end{tabular}

\section{Conclusion}

In the era of signal processing, wavelets are the novel tool for the analysis of its individual picture element without disturbing its originality and it has enriched with significant progress in the recent decade. In the idea of Image compression, wavelets are grabbing a noticeable impact. In this paper the image is compressed utilizing Haar wavelet and color space model and reconstruction of image is finished by utilizing reverse process. Results appear in Table 1. The first image is compressed by 70 percent with no degradation of image quality,the extent of the reconstructed image is same and in addition unique image.

\section{Future scope}

This can be used for transmitting images in low power, less bandwidth wireless networks. The compression technique is applicable for both homo dimensional image and hetero dimensional image.

\section{Acknowledgment}

The authors like to express their thanks to the department of ECE, S.J.C.I.T for their support and encouragement during this work.

\section{References}

[1] T. M. Cover and J. A. Thomas, Elements of Information Theory, 2nd ed. John Wiley and Sons, Jul. 2006.

[2] D. L. Donoho, M. Vetterli, R. A. Devore, and I. Daubechies, "Data compression and harmonic analysis," IEEE Transaction on

[3] Information Theory, vol. 44, pp. 2435 -2476, Oct. 1998.

[4] Z. Liu, Context-Based and Perceptual-Based Wavelet Image Compression with Application to JPEG2000, Ann Arbor, Mich.:

[5] A Haar, "Zur theorieder orthogonalen funktionensysteme," Mathematische Annalen, vol. 69, no. 3, pp. 331-371, 1910.

[6] Ph. W. Besslich and E. A. Trachtenberg, "The sign transform: An invertible non-linear transform with quantized coefficients,"

[7] Theory and Application of Spectral Technique, C. Moraga, Ed. University Dortmund Press, Oct. 1988, pp. 150-160.

[8] Sridhar C S, Dr.Mahadevan.G, S.K.Khadar basha , Design And Implementation Of A Color Space Conversion Module Using Open-

[9] Computer Vision, vol. 12-special issue. Journal of Advanced Research in Dynamical and Control Systems, Scopus SJR pp. 2321-2329.ISSN 1943-023X, Dec. 2017

[10] T.Padmapriya and V.Saminadan, "Utility based Vertical Handoff Decision Model for LTE-A networks", International Journal of Computer Science and Information Security, ISSN 1947-5500, vol.14, no.11, November 2016.

[11] S.V.Manikanthan and K.Baskaran "Low Cost VLSI Design Implementation of Sorting Network for ACSFD in Wireless Sensor Network", CiiT International Journal of Programmable Device Circuits and Systems,Print: ISSN 0974 - 973X \& Online: ISSN 0974 - 9624, Issue: November 2011, PDCS112011008.

[12] Rajesh, M., and J. M. Gnanasekar. \&quot; CONGESTION CONTROL USING AODV PROTOCOL SCHEME FOR WIRELESS AD-HOC NETWORK.\&quot; Advances in Computer Science and Engineering 16.1/2 (2016): 19.

[13] S.V.Manikanthan and V.Rama"Optimal Performance Of Key Predistribution Protocol In Wireless Sensor Networks" International Innovative Research Journal of Engineering and Technology, ISSN NO: 2456-1983,Vol-2, Issue -Special March 2017. 